\title{
Migraine-Associated Otalgia: An Underappreciated Entity
}

\author{
Sarah Sussman ${ }^{1}$, Zachary Zimmerman $^{2}$, Taylor Chishom ${ }^{3}$, Lauren Reid ${ }^{4}$, and Mohammad Seyyedi ${ }^{1}$ \\ ${ }^{1}$ Department of Otolaryngology, Medical College of Georgia Augusta University, Augusta, GA, USA \\ ${ }^{2}$ Department of Otolaryngology, Northwestern University, Chicago, IL, USA \\ ${ }^{3}$ Department of Plastic Surgery, Virginia Commonwealth University Health, Richmond, VA, USA \\ ${ }^{4}$ Department of Otolaryngology, University of Tennessee Health Science Center, Memphis, TN, USA
}

$\begin{array}{ll}\text { Received } & \text { August 18, 2021 } \\ \text { Revised } & \text { November 10, 2021 } \\ \text { Accepted } & \text { November 26, 2021 }\end{array}$

Address for correspondence

Mohammad Seyyedi, MD

Department of Otolaryngology,

Medical College of Georgia

Augusta University,

1120 15th ST, BP-4109,

Augusta, GA 30912, USA

Tel +1-706-721-6100

Fax +1-706-721-0112

E-mail mseyyedi@augusta.edu
Background and Objectives: Otalgia can be primary/otogenic or secondary as a referred pain from another site, which can be difficult to establish owing to various causes and the complex innervation of the ear. In our center, we observed a large group of patients with unexplained otalgia that had a higher prevalence of migraine. We hypothesized that migraine may cause secondary otalgia. This study then aimed to determine the prevalence of migraine-associated otalgia and evaluate the efficacy of migraine treatment. Subjects and Methods: This 2-year retrospective study was conducted at a busy otology clinic. Patients were identified using diagnostic codes corresponding to otalgia. The prevalence of migraine-associated otalgia was determined, and the efficacy of migraine treatment was evaluated in these patients. The interventions included prophylactic and abortive migraine treatments. Statistical analysis was conducted to compare between the pre- and post-treatment symptoms. Results: A total of 208 patients with otalgia were identified. Sixty-four out of ninety patients with unexplained otalgia met the criteria for migraine; of them, 30 patients had an adequate follow-up and were thus included in the evaluation of treatment efficacy. Otalgia improved in $87 \%$ of the patients who received migraine treatment. After treatment, the mean pain score and headache frequency significantly decreased from 7 to 2 and from 27 to 9 days per month, respectively $(p<0.001)$. Conclusions: Migraine should be considered as a source of secondary otalgia, and patients should receive treatment as they often respond to migraine treatment. J Audiol Otol 2022;26(2):90-96

Keywords: Otalgia; Migraine; Migraine disorders; Secondary otalgia.

\section{Introduction}

Otalgia is a common chief complaint in both primary care settings and the emergency room. More than 580,000 emergency departmeat visits in 2009-2011 in the United States were attributed to otalgia [1]. Despite its high prevalence, the diagnosis and treatment of otalgia can be very challenging due to the broad range of etiologies. Otalgia can be primary due to a pathology within the ear or secondary as a referred pain from another site. Overall, primary causes tend to be more common. One clinical study found that roughly $70 \%$ of patients in their practice were diagnosed with an otogenic etiology for their otalgia, and about $30 \%$ were diagnosed with a secondary etiology

This is an Open Access article distributed under the terms of the Creative Commons Attribution Non-Commercial License (https://creativecommons.org/licenses/by-nc/4.0/) which permits unrestricted non-commercial use, distribution, and reproduction in any medium, provided the original work is properly cited.
[2]. Men tend to have a primary cause, while women tend to have a secondary cause $[2,3]$. The most common primary causes of otogenic otalgia are chronic and acute otitis media, external auditory canal infection and ear trauma [2]. The etiology of secondary otalgia can be often difficult to establish due to various causes and the complex innervation of the ear. The ear receives sensory innervation from both cranial and spinal nerves and involvement of these nerves in different pathologies may result in secondary otalgia. The trigeminal, facial, glossopharyngeal and vagus nerves (CN V, VII, IX, X) are the cranial nerves and spinal nerves include the lesser occipital (C2, C3) and greater auricular (C2, C3) nerves [4]. Common secondary causes include temporomandibular joint disorder (TMJD), pharyngotonsillitis, dental issues, or even the sentinel symptom of a head and neck malignancy [2,3].

In our tertiary referral center, we have experienced a large percentage of patients presenting with otalgia that their pain 
could not be attributed to any primary or secondary reason even with thorough work-up including complete history, head and neck exam which may include endoscopic nasopharyngolaryngologic exam, and imaging tests when warranted such as MRI, CT, and ultrasound. In some instances, patients were referred to our colleagues in other specialties; however, no explanation for otalgia was discovered.

In the past decade we learned about the association between unexplained otologic symptoms such as dizziness with migraine headaches which is now called vestibular migraine. It was shown that the convergence of the trigeminal-vestibulocochlear system can result in dizziness [5]. Accordingly, we hypothesize that migraine may play a role in some otalgia cases with unknown source.

Convergence is believed to be a phenomenon in which a single second-order neuron receives nociceptive input from multiple sites, and the brain is unable to localize the correct primary stimulus [6]. It has been shown that migraine pain arises from the activation of meningeal perivascular afferents which are innervated by trigeminal nerve (CN V) $[7,8]$. The trigeminal nerve also gives off numerous branches throughout the ear including those that provide sensation to the anterosuperior pinna, external auditory canal and the lateral tympanic membrane [6]. This viscerosomatic convergence of meningeal and extracranial cutaneous afferents can explain the extracranial symptoms of migraine such as allodynia in the territory of trigeminal nerve [9]. This may explain the role of migraine in secondary otalgia, though otologic migraine-associated symptoms have not been characterized in the literature as well as other migraine symptomology. Perhaps this subset of patients does not present to neurologists or neurologists do not associate otalgia with migraine [10].

In our tertiary care center, we observed that many of our patients with an unknown source of otalgia have a higher prevalence of migraine that often goes unrecognized by both the patients and the referring providers. Likewise, Kuttila et al. [11] found that the frequency of headache is higher in patients with otalgia. On the other hand Anttila et al. [12] reported that patients with migraine are more likely to have otalgia compared with patients suffering from other types of headache. The purpose of this study aims to more precisely describe the association between otalgia and migraine and to propose effective therapy for this underrecognized patient population.

\section{Subjects and Methods}

The Institutional Review Board at Augusta University Medical Center approved this retrospective study of patients from an otologic practice at a busy tertiary care center from August
2016 to October 2018 (No. 1370510-5). The informed consent was waived. All patients with ICD-10 codes corresponding to otalgia were identified from the electronic medical record system. Patient charts were reviewed for detailed medical history, age, sex, duration of otalgia, characteristics of pain, laterality, triggers, frequency and severity of otalgia pre- and posttreatment, tinnitus, hearing loss, ear fullness, vertigo, and prior ear surgery, physical exam findings, imaging studies, audiologic findings, and detailed headache history and associated symptoms including nausea, vomiting, sensitivity to sound, light, and cold. The reason for otalgia was determined based on the collected data (history, physical exam and paraclinical tests) and the percentages of different etiologies causing otalgia were calculated. Patients were classified in 3 major categories: 1- primary otalgia, 2- secondary otalgia with known reason, and 3- secondary otalgia with unknown reason. Primary otalgia patients were identified as having a source of otalgia within the ear itself such as otitis externa. Secondary otalgia patients with known source were defined as having ear pain without any identifiable source in the ear but with a known pathology outside the ear such as TMJD. Patients with secondary otalgia with unknown reason were defined as patient with no known source for otalgia inside or outside the ear itself where the symptoms, physical exam and paraclinical tests did not point to a specific cause.

To study the association between otalgia and migraine, those with the known primary and secondary etiologies such as acute and chronic otitis media as well as TMJD were excluded from the study. From this recent group, the patients who met the following criteria were included for the study of the efficacy of treatment: 1- patients diagnosed with otalgia of unknown origin, 2- positive history of migraine headaches meeting the criteria defined by the International Headache Society [13], and 3- adequate follow-up, defined as a minimum followup period of 3 months and correspondence of how patients were feeling.

These patients were treated similar to migraineurs $[14,15]$; Patients with 8 and more otalgia days per month were treated by abortive and preventive (prophylactic) medicine and patients with $1-7$ otalgia days were treated by abortive medicine only. All patients were given a list of migraine trigger foods and were asked to avoid those foods. They were instructed to keep a diary of their symptoms. We used a stepwise approach to pharmacotherapy if escalation was warranted (Fig. 1). We started patients with one of the NSAIDs as abortive treatment as well as one of the 1st line prophylactic medications such as verapamil, nortriptyline, and topiramate or 2 nd line preventive medication such as propranolol where it was indicated. Botox and alternative medications like gab- 
apentin were tried after failure of three of the above preventive medications and/or intolerable side effects from the traditional migraine therapies.

Evaluation of the patients' response to treatment was done similar to the evaluation of the efficacy of migraine headaches treatment. Patients were given a symptom log and they were asked to record their pain scores $(0-10)$ and earaches days per month (0-31). A successful treatment was defined by at least a $50 \%$ reduction in their pain score average in a month and/or earache days. Pretreatment scores were compared with posttreatment scores. All statistical analyses were performed using SPSS statistical package version 27 (IBM Corp., Armonk, NY, USA). Descriptive statistics were used for demographic calculations. A Wilcoxon signed-rank test was used for the comparisons of nonparametric data and t-test for parametric data. Statistical significance was defined as alpha level less than 0.05 and confidence interval of $95 \%$ in all statistical tests.

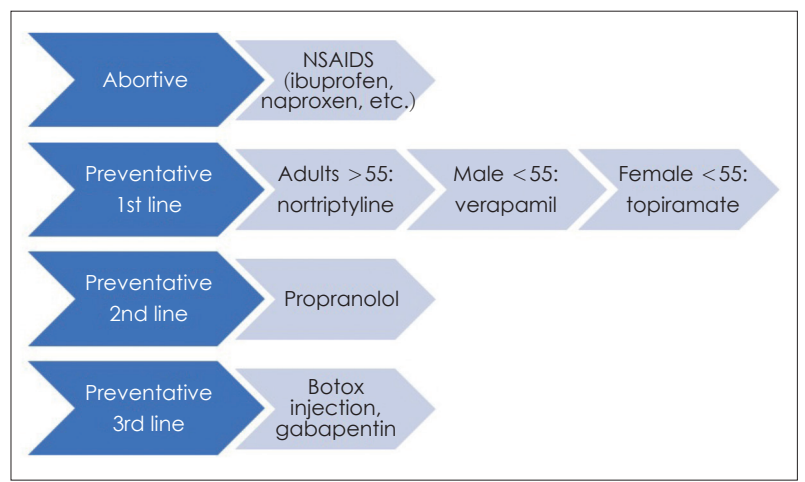

Fig. 1. Treatment algorithm for migrainious otalgia.

\section{Results}

\section{Patient demographics \& characteristics}

A total of 208 patients with otalgia were identified, 159 females and 49 males (Table 1). Otologic primary pathology was the source of otalgia in 64 patients (31\%) and 54 patients $(26 \%)$ had secondary, referred otalgia with known pathology. Like previous studies, the most common primary and secondary otalgia cases were acute and chronic otitis media and temporomandibular joint arthralgia. In the remaining 90 patients (43\%) no explanation was found for otalgia. 85 out of 90 patients $(94 \%)$ had otalgia with headaches in which 64 patients with unexplained otalgia (71\%) met the International Headache Society (IHS) criteria for migraine headaches. The prevalence of migraine (71\%) in patients with unexplained otalgia is significantly higher than the general population in the United States in 2015 (15.3\%), reported by Burch et al. [16] $\left(\chi^{2}=\right.$ 210.39 , $p$-value is $<0.001)$. As can be seen in Table 1 , otalgia is more common in females and white ethnicity in all the groups of otalgia. The female to male ratio in patients with migrainous otalgia (4.8:1) is higher than the female to male ratio in non-headache otalgia group $(2.6: 1)$, but didn't reach a significant level $\left(\chi^{2}=2.51, p\right.$-value $\left.=0.110\right)$.

Thirty patients out of sixty four patients with migraine headaches and unexplained otalgia (migrainous otalgia group) had adequate follow-up and received the treatment similar to migraine headaches and were included in the final analysis for efficacy of treatment. There were 25 females and 5 males in this group and the female to male ratio was 5:1. The age range was from 22 to 93 years with a mean of 52.6 years. Demographics are summarized in Table 1 . The age and female-to-

Table 1. Patient demographics \& characteristics

\begin{tabular}{|c|c|c|c|c|}
\hline & All patients & $\begin{array}{c}\text { All patients w/otalgia w/o } \\
\text { headache }\end{array}$ & $\begin{array}{l}\text { All migrainous } \\
\text { otalgia }\end{array}$ & $\begin{array}{c}\text { Migrainous } \\
\text { otalgia } \mathrm{w} / \text { treatment }\end{array}$ \\
\hline Age (yr) & $48.5 \pm 17.7$ & $47.00 \pm 18.63$ & $51.51 \pm 15.47$ & $52.6 \pm 18.9$ \\
\hline \multicolumn{5}{|l|}{ Sex } \\
\hline Female & 159 & 89 & 53 & 25 \\
\hline Male & 49 & 34 & 11 & 5 \\
\hline Total & 208 & 123 & 64 & 30 \\
\hline Sex ratio (F:M) & $3.2: 1$ & $2.6: 1$ & $4.8: 1$ & $5: 1$ \\
\hline \multicolumn{5}{|l|}{ Race } \\
\hline White & $120(57.7)$ & $78(63.4)$ & $28(43.7)$ & $20(66.7)$ \\
\hline Black & $70(33.6)$ & $31(25.2)$ & $34(53.1)$ & $8(26.6)$ \\
\hline Other & $18(8.7)$ & $14(11.4)$ & $2(3.1)$ & $2(6.7)$ \\
\hline \multicolumn{5}{|l|}{ Laterality } \\
\hline Right & $86(41.3)$ & $51(41.5)$ & $32(50.0)$ & $9(30.0)$ \\
\hline Left & 67 (32.3) & $39(31.7)$ & $16(25.0)$ & $9(30.0)$ \\
\hline Bilateral & $55(26.4)$ & $33(26.8)$ & $16(25.0)$ & $12(40.0)$ \\
\hline
\end{tabular}

Data are presented as mean \pm standard deviation or $\mathrm{n}(\%)$ 
male ratio in this migrainous otalgia subgroup $(n=30)$ were not significantly different from the main migrainous otalgia group $(\mathrm{n}=64)$ ( $p$-values $=0.208$ and 0.784 , respectively).

\section{Symptom profile and associated symptoms in the treatment group}

The median duration of symptoms before diagnosis was 3.5 months. Triggerability of otalgia was seen in $47 \%$ of patients. Valsalva, exertion, head movement, change in body position, sound, bright lights, cold, change of barometric pressure and altitude were such documented triggers. Symptoms were bilateral in $40 \%$ of patients ( $n=12$ ). Otalgia was the chief complaint in $93 \%(n=28)$ of the patients. Ear pain was characterized as continuous by $30 \%(\mathrm{n}=9)$, sharp by $13 \%(\mathrm{n}=4)$, throbbing by $10 \%(\mathrm{n}=3)$, and dull by $7 \%(\mathrm{n}=2)$. Other subjective characteristics of pain included burning, scalding, pulsating, swishing, and popping. Aural fullness/pressure was reported in $20 \%(\mathrm{n}=6)$. Tinnitus was reported in $40 \%(\mathrm{n}=12)$ and vertigo in $23 \%$ ( $n=7$ ) (Table 2 and Fig. 2).

Migraine associated symptoms were also recorded. Both phonophobia and photophobia were reported by $43 \%$ of patients $(n=13)$. Nausea and/or vomiting were reported in $10 \%$ of patients. The duration of ear pain ranged from 1 week to 3 years with a mean duration of 3.5 months, and the duration of headache history ranged from 1 week to 30 years.

\section{Treatment}

Similar to migraine treatment, patients with 8 and more otalgia days per month were treated by preventive and abortive medications and patients with 1-7 otalgia days were treated by abortive medicine only. All patients were given a list of potential trigger foods and were asked to avoid them. Out of the thirty patients studied, twenty-six patients were treated with migraine prophylaxis consisting of nortriptyline, propranolol, topiramate, gabapentin, or verapamil. Four were treated conservatively only with diet modifications per their request. Two received botox injections per PHASE III Research Evaluating Migraine Prophylaxis Therapy (PREEMPT) protocol, after failing 2-3 medications above, which are approved for chronic intractable migraine (at least 15 days per month lasting 4 hours per day) [17]. Fig. 1 shows the algorithm used to treat migrainous otalgia which was based on the known treatment of migraine and the patient's health status, risk factors, and medical history.

Improvement of otalgia, which was defined by at least 50\% reduction in average pain score or headache days per month, occurred in $87 \%$ of patients $(n=26)$ and $57 \%(n=17)$ reported complete resolution of their symptoms. Two patients did not respond to the treatment, and two patients worsened after fail-
Table 2. Reported otalgia symptoms in our patient population $(n=30)$

\begin{tabular}{lc}
\hline & Value \\
\hline Laterality & $18(60)$ \\
Unilateral & $12(40)$ \\
Bilateral & $14(47)$ \\
Triggerability & $6(20)$ \\
Positional change & $5(17)$ \\
Sound or light & $3(10)$ \\
Valsalva & \\
Pain characteristics & $9(30)$ \\
Constant & $6(20)$ \\
Aural fullness & $4(13)$ \\
Sharp & $3(10)$ \\
Throbbing & $2(7)$ \\
Dull & $6(20)$ \\
Other & \\
Associated symptoms & $12(40)$ \\
Tinnitus & $10(33)$ \\
Hearing loss & $7(23)$ \\
Vertigo &
\end{tabular}

Data are presented as $\mathrm{n}(\%)$

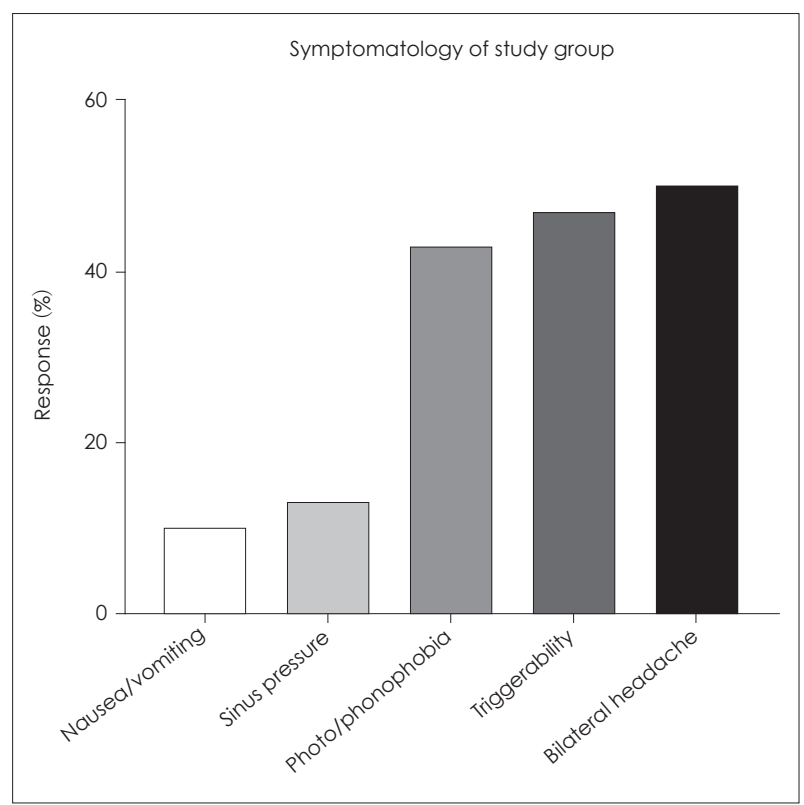

Fig. 2. Symptomatology of treatment study group.

ing 2 to 3 classes of medications (Table 3). Prior to receiving therapy, $63 \%(n=19)$ of our population had constant ear pain. After treatment, $58 \%(\mathrm{n}=11)$ of these 19 patients reported complete resolution of their pain. Median follow-up time was 6.5 months. The mean number of otalgia days per month (out of 30 days) significantly decreased from 27 pre-treatment to 9 post-treatment ( $p$-value $<0.001$ ). The mean pre-treatment pain score was 7 and significantly decreased to an average of 
Table 3. Treatment efficacy based on patient questionnaire, broken into primary and secondary treatment medications

\begin{tabular}{lcccc}
\hline \multirow{2}{*}{ Medication } & \multicolumn{2}{c}{$\begin{array}{c}\text { Primary } \\
\text { treatment }\end{array}$} & $\begin{array}{c}\text { Secondary } \\
\text { treatment }\end{array}$ \\
\cline { 2 - 5 } & $\mathrm{n}$ & $\begin{array}{c}\text { Improved } \\
(\%)\end{array}$ & $\mathrm{n}$ & $\begin{array}{c}\text { Improved } \\
(\%)\end{array}$ \\
\hline Tricyclic antidepressant & 11 & 91 & 7 & 57 \\
Topiramate & 6 & 50 & 1 & 100 \\
Propranolol & 4 & 80 & 1 & 100 \\
Verapamil & 4 & 74 & 1 & 0 \\
Gabapentin & 1 & 100 & 0 & 0 \\
Botox Injections* & & & 2 & 100 \\
\hline
\end{tabular}

*onabotulinumtoxin A injections administered per PHASE III Research Evaluating Migraine Prophylaxis Therapy (PREEMPT) protocol

2 post-treatment ( $p$-value $<0.001$ ). These scores were extrapolated from patients' otalgia journal kept daily during the treatment interval ( 0 being no pain and 10 being the worst pain).

\section{Discussion}

In the current literature, migraine pathophysiology and treatment data have been dominantly produced by neurologists as it was thought to be a purely neurologic disease, but with the light shone from other perspectives, we know that migraine can present with many different symptoms other than headache involving other specialties including otolaryngology [10]. It is conceivable for an otolaryngologist presented with migrainous otalgia, to easily miss the diagnosis and attribute the symptoms to a primary ear or sinus condition [10]. Many studies documenting common causes for referred otalgia in the clinical setting do not examine migraine as a potential cause [18-20]. For example, in a study of origins of referred otalgia in a tertiary setting, 150 out of 450 patients had referred otalgia in which roughly $5 \%$ of patients ( 7 out of 150 ) had an unknown origin for their otalgia and none was attributed to migraines. Unfortunately, there are no standardized clinical criteria for diagnosing migraine associated otalgia and no easily discernable physical exam or radiologic findings that make the diagnosis of other causes of referred otalgia more straight-forward.

Headache and dizziness are the only two typical migraine symptoms in the head and neck that are defined by clinical criteria (the IHS for headache and Nehauser's for vestibular migraine) $[10,13,21]$. Various other head and neck manifestations can occur outside of the acute headache but are believed to result from the same pathophysiology process of migraines [10]. These symptoms include sinus pressure, nasal congestion, and runny nose [22]. Referred migraine pain is common throughout the head and neck, and as many as $80 \%$ of patients with a "sinus" headache are diagnosed as fulfilling
IHS migraine criteria at time of screening [23]. Otologic symptoms of migraine include ear fullness and pressure, ear pain, sound sensitivity, and tinnitus [24,25]. Sabra et al. [10] described ear pain and fullness as the most common non-dizzy migrainous ear symptoms. "50 patients $(46.3 \%)$ presented for an atypical chief complaint out of which $34.2 \%$ were ear symptoms (mostly ear pain and fullness) and $14.8 \%$ nasal symptoms (mostly nasal congestion and sinus pressure) and 2.7\% had an ear and nose atypical chief complaint." According to Schulz et al. [22], 38.2\% of patients with positive migraine screening also reported unusual ear sensitivity and 38\% experienced ear pressure. Teixido et al. [25] found that $77 \%$ of their patients presenting with otalgia had a concordant headache history. All these data show the importance of the study of other manifestations of migraine specially in the head and neck area where they are dominantly innervated by trigeminal nerve.

Migrainous otalgia has been previously described only sparingly throughout the literature. One other paper was identified in our literature review that described the experience of treating patients with referred otalgia with traditional migraine therapy [25].

In agreement with the criteria utilized by Teixido et al. [25], we define migraine-associated otalgia or migrainous otalgia as otalgia in the setting of:

1) The absence of primary otogenic problem such as otitis media and secondary otalgia associated with non-otogenic problem such as temporomandibular joint dysfunction, or upper aerodigestive disorder.

AND 1 or more of the following:

1) IHS defined migraine headaches and its subtypes,

2) triggerability by migraine triggers,

3) response to migraine therapy.

In our tertiary medical center, migrainous otalgia was found in 64 patients out of 208 patients, which was as common as primary otalgia $(n=64)$. This high rate of migrainous otalgia can be due to the difficulty of diagnosis of such cases in the community by the primary care providers or general otolaryngologists and the resultant higher rate of referring to higher level of care. The female to male ratio in our migrainous otalgia group was 4.8:1 which was lower than this ratio in Teixido et al.'s study [25] (12:1) and was greater than the reported 3.3:1 ratio in the adult migraine population [26], though none of these differences reached a significant level $\left(\chi^{2}=1.1431\right.$, $p$-value $=0.285$ and $\chi^{2}=1.3489, p$-value $=0.245$, respectively) .

According to Olsson [27], "if a patient has migraine, but also has significant cochlear and/or vestibular dysfunction, the migraine syndrome and the peripheral organ must both be treated." Our study is among the earliest to evaluate treat- 
ment outcomes in patients with otalgia and migraine headaches by using migraine therapy. We propose treatment of migraine associated otalgia with migraine therapy.

Our study reveals that otalgia of unknown source in patients with history of migraine can be successfully treated with traditional migraine therapy. Our results echo the findings of Teixido et al.'s study [25] which showed $92 \%$ of 26 patients showed more than a $50 \%$ reduction in symptom scores with migraine treatment. In our study, $87 \%$ had significant improvement and $57 \%$ of patients experienced complete resolution of their earache after treatment. Our sample size was modestly larger at thirty patients even after excluding thirty four patients due to inadequate follow-up. Based on our experience, we created a treatment algorithm (Fig. 1). To follow this algorithm, one should be aware of the side effects and contraindications of these medications. For example, propranolol is not recommended for diabetic and asthmatic patients and those with heart failure.

As with any retrospective chart review, a limitation is reliance on correct documentation and diagnosis tracking in the electronic medical record. Another limitation in our study is patient loss to follow-up. We had sixty four patients with unexplained otalgia who met IHS criteria for migraine and only thirty patients who had sufficient follow-up were analyzed for the efficacy of the treatment. However, the mean age and gender distribution were not statistically different between the treatment study group and all migrainous otalgia group which made this loss of follow up less detrimental. The other limitation was having more than one reason for otalgia in some patients where detailed history, physical exam and response to the different treatments were used to determine the main reason of otalgia.

Due to markedly common presentation, it is necessary to explore the frequency of stress symptoms, bruxism, and recurrent neck pain in patients with secondary otalgia [11] This project is an introduction to a cohort research project to study the migrainous otalgia and the outcome of the treatment.

In conclusion, migraine should be considered in the differential diagnosis of otalgia. In those with absence of other etiologies and with a history of migraine headaches, migrainous otalgia is likely. As our findings demonstrated, these patients often respond to known abortive and prophylactic migraine medications.

\section{Acknowledgments \\ None}

\section{Conflicts of interest}

The authors have no financial conflicts of interest.

\section{Author Contributions}

Conceptualization: Mohammad Seyyedi, Zachary Zimmerman. Data curation: all authors. Formal analysis: Zachary Zimmerman, Mohammad Seyyedi, Sarah Sussman. Methodology: all authors. Supervision: Mohammad Seyyedi. Validation: Mohammad Seyyedi, Zachary Zimmerman. Writing — original draft: Sarah Sussman, Zachary Zimmerman, Mohammad Seyyedi. Writing — review \& editing: all authors. Approval of final manuscript: all authors.

\section{ORCID iDs}

Sarah Sussman

Zachary Zimmerman

Taylor Chishom

Lauren Reid

Mohammad Seyyedi

https://orcid.org/0000-0001-7108-5118

https://orcid.org/0000-0001-7948-5503

https://orcid.org/0000-0003-3529-7038

https://orcid.org/0000-0001-7536-5248

https://orcid.org/0000-0002-3120-8459

\section{REFERENCES}

1) Kozin ED, Sethi RK, Remenschneider AK, Kaplan AB, Del Portal DA, Gray ST, et al. Epidemiology of otologic diagnoses in United States emergency departments. Laryngoscope 2015;125:1926-33.

2) Kim SH, Kim TH, Byun JY, Park MS, Yeo SG. Clinical differences in types of otalgia. J Audiol Otol 2015;19:34-8.

3) Taziki MH, Behnampour N. A study of the etiology of referred otalgia. Iran J Otorhinolaryngol 2012;24:171-6.

4) Szymanski A, Geiger Z. Anatomy, head and neck, ear. Treasure Island: StatPearls;2020.

5) Espinosa-Sanchez JM, Lopez-Escamez JA. New insights into pathophysiology of vestibular migraine. Front Neurol 2015;6:12.

6) Norris CD, Koontz NA. Secondary otalgia: referred pain pathways and pathologies. AJNR Am J Neuroradiol 2020;41:2188-98.

7) Olesen J, Burstein R, Ashina M, Tfelt-Hansen P. Origin of pain in migraine: evidence for peripheral sensitisation. Lancet Neurol 2009; 8:679-90.

8) Strassman A, Mason P, Moskowitz M, Maciewicz R. Response of brainstem trigeminal neurons to electrical stimulation of the dura. Brain Res 1986;379:242-50.

9) Ramachandran R, Yaksh TL. Therapeutic use of botulinum toxin in migraine: mechanisms of action. Br J Pharmacol 2014;171:4177-92.

10) Sabra O, Muhammad Ali M, Al Zayer M, Altuwaijri S. Frequency of migraine as a chief complaint in otolaryngology outpatient practice. Biomed Res Int 2015;2015:173165.

11) Kuttila SJ, Kuttila MH, Niemi PM, Le Bell YB, Alanen PJ, Suonpää JT. Secondary otalgia in an adult population. Arch Otolaryngol Head Neck Surg 2001;127:401-5.

12) Anttila $P$, Metsähonkala L, Mikkelsson $M$, Helenius H, Sillanpää M. Comorbidity of other pains in schoolchildren with migraine or nonmigrainous headache. J Pediatr 2001;138:176-80.

13) Headache Classification Committee. Headache Classification Committee of the International Headache Society (IHS) the international classification of headache disorders, 3rd edition. Cephalalgia 2018; 38:1-211.

14) Silberstein S, Tfelt-Hansen P, Dodick DW, Limmroth V, Lipton RB, Pascual J, et al. Guidelines for controlled trials of prophylactic treatment of chronic migraine in adults. Cephalalgia 2008;28:484-95.

15) Dodick DW. Migraine. Lancet 2018;391:1315-30.

16) Burch R, Rizzoli P, Loder E. The prevalence and impact of migraine and severe headache in the United States: figures and trends from government health studies. Headache 2018;58:496-505.

17) Tepper D. Onabotulinumtoxin [corrected] A (Botox). Headache 2014;54:787-8.

18) Anwar K, Khan S, Shahabi I, Niazi ZB. The frequency of involvement of head \& neck sites in referred otalgia - An experience at a 
tertiary care hospital. Pak J Med Sci 2019;35:1138-42.

19) Finnikin S, Mitchell-Innes A. Recurrent otalgia in adults. BMJ 2016; 354:i3917.

20) Harrison E, Cronin M. Otalgia. Aust Fam Physician 2016;45:493-7.

21) Neuhauser $H$, Leopold $M$, von Brevern $M$, Arnold G, Lempert T. The interrelations of migraine, vertigo, and migrainous vertigo. Neurology 2001;56:436-41.

22) Schulz KA, Esmati E, Godley FA, Hill CL, Monfared A, Teixido M, et al. Patterns of migraine disease in otolaryngology: a CHEER network study. Otolaryngol Head Neck Surg 2018;159:42-50.

23) Schreiber CP, Hutchinson S, Webster CJ, Ames M, Richardson MS, Powers C. Prevalence of migraine in patients with a history of selfreported or physician-diagnosed "sinus" headache. Arch Intern Med
2004;164:1769-72.

24) Neff BA, Staab JP, Eggers SD, Carlson ML, Schmitt WR, Van Abel $\mathrm{KM}$, et al. Auditory and vestibular symptoms and chronic subjective dizziness in patients with Ménière's disease, vestibular migraine, and Ménière's disease with concomitant vestibular migraine. Otol Neurotol 2012;33:1235-44.

25) Teixido M, Seymour P, Kung B, Lazar S, Sabra O. Otalgia associated with migraine. Otol Neurotol 2011;32:322-5.

26) Lipton RB, Bigal ME, Diamond M, Freitag F, Reed ML, Stewart WF; AMPP Advisory Group. Migraine prevalence, disease burden, and the need for preventive therapy. Neurology 2007;68:343-9.

27) Olsson JE. Neurotologic findings in basilar migraine. Laryngoscope 1991;101:1-41. 